\title{
Effects of the Tibetan Plateau Crustal Structure on the Inversion of Water Trend Rates Using Simulated GRACE/GPS Data
}

\author{
Hansheng Wang ${ }^{1, *}$, Longwei Xiang ${ }^{1,2}$, Patrick Wu ${ }^{3}$, Holger Steffen ${ }^{3}$, Lulu Jia ${ }^{1,2,4}$, \\ Liming Jiang ${ }^{1}$, and Qiang Shen ${ }^{1}$ \\ ${ }^{1}$ State Key Laboratory of Geodesy and Earth's Dynamics, Institute of Geodesy and Geophysics, \\ Chinese Academy of Sciences, Wuhan, China \\ ${ }^{2}$ College of Earth Sciences, University of Chinese Academy of Sciences, Beijing, China \\ ${ }^{3}$ Department of Geoscience, University of Calgary, Calgary, Canada \\ ${ }^{4}$ National Earthquake Infrastructure Service, Beijing, China
}

Received 1 May 2012, accepted 21 September 2012

\begin{abstract}
The crustal structure under the Tibetan Plateau is quite different from that given by the commonly used Preliminary Reference Earth Model (PREM, Dziewonski and Anderson 1981). We investigate the effects of such differences on inversion results of water trend rates in the area using simulated GRACE (Gravity Recovery and Climate Experiment) and GPS data. When using simulated GRACE data for the inversion, the effects of crustal differences are negligible, confirming the validity of using PREM for the inversion of water trend rates from current and even follow-on GRACE data. However, when using simulated GPS data, effects of crustal differences are very prominent suggesting that an Earth model with a realistic crustal structure instead of PREM should be used for this case.
\end{abstract}

Key words: Tibetan Plateau, GPS, GRACE, Crustal structure, Water trend

Citation: Wang, H., L.Xiang, P. Wu, H. Steffen, L. Jia, L. Jiang, and Q. Shen, 2013: Effects of the Tibetan Plateau crustal structure on the inversion of water trend rates using simulated GRACE/GPS data. Terr. Atmos. Ocean. Sci., 24, 505-512, doi: 10.3319/TAO.2012.09.21.01(TibXS)

\section{INTRODUCTION}

Space-borne gravimetry and GPS techniques play increasingly important roles in the determination of surface water storage (denoted by equivalent water thickness, EWT) changes and the trend rates especially for the high mountain areas like Tibetan Plateau. In this work, we study how Tibetan Plateau crustal structure affect the results for the inversion of water trend rates based on simulated GRACE/ GPS data.

Since the launch of the twin-satellites of Gravity Recovery and Climate Experiment (GRACE) in 2002, the Earth's time-varying gravity changes can be precisely monitored on a global scale and the data are successfully used to estimate, based on the method outlined by Wahr et al. (1998), water storage changes and trends both on land (e.g., Tibetan Plateau, Yangtze River watershed, Ganges River, Amazon basin, Congo Basin, polar areas) and ocean areas at about

\footnotetext{
* Corresponding author

E-mail:whs@asch.whigg.ac.cn
}

300 - $400 \mathrm{~km}$ resolution (e.g., Tapley et al. 2004; Wang et al. 2007; Chen et al. 2009; Leuliette and Miller 2009; Rodell et al. 2009; Matsuo and Heki 2010). In this regard, it is well known that the gravity changes observed by GRACE are not only due to the direct attraction from surface mass changes but also from internal mass redistribution induced by the elastic loading of surface loads. Furthermore, the results of the inversion of water storage changes based on GRACE data can rely in part on the selected Earth model as it delivers important input parameters for the method used by Wahr et al. (1998). On the other hand, the vertical displacements induced by the elastic loading can be observed with GPS, and these displacements also allow the inversion of water storage changes.

It should be noted that the Preliminary Reference Earth Model (PREM, Dziewonski and Anderson 1981) is usually used to compute the load Love numbers (e.g., Farrell 1972; Han and Wahr 1995; Wang et al. 1996; Guo et al. 2001; Van Dam et al. 2002) in nearly all the related studies 
(e.g., Wang et al. 2007; Matsuo and Heki 2010; Jacob et al. 2012) because it gives a globally averaged elastic structure. However, the crustal elastic structure in the Crust 2.0 model (Laske et al. 2011) which has a higher depth resolution is markedly different from that given by PREM. In the Tibetan Plateau, the crust is found to be the thickest in the world. In this paper, we investigate the effects of the regional crustal structure of the Tibetan Plateau on the inversion of water trends based on simulated GRACE and GPS data.

In the next section, the Earth models and related load Love numbers used are described. The formulas for the simulated inversion are derived in section 3. Simulated GRACE and GPS data are shown in section 4. The computational results are presented and discussed in section 5. Finally, the conclusions are summarized in section 6 .

\section{EARTH MODELS AND LOAD LOVE NUMBERS}

For PREM, the globally averaged density, P-Wave velocity and S-Wave velocity are shown in Fig. 1. In order to investigate the effects of the crustal differences on the simulated inversion of water trend rates in the area, we devise a modified and more realistic model MQ-PREM which is based on the PREM model below the crust and the average crustal structure given by Crust 2.0 as shown in Fig. 1. It can be seen that within a depth range of $80 \mathrm{~km}$, the density, the two wave velocities, and thus the elastic Lame constants are much less than those given by PREM. Therefore this crust for the Tibetan Plateau could be more easily deformed than a crust based on PREM.

We use the variable transformation method by Wang et al. (2012) to compute the two load Love numbers $\left(h_{n}, k_{n}\right)$ for the PREM and MQ-PREM Earth models (Fig. 2). As

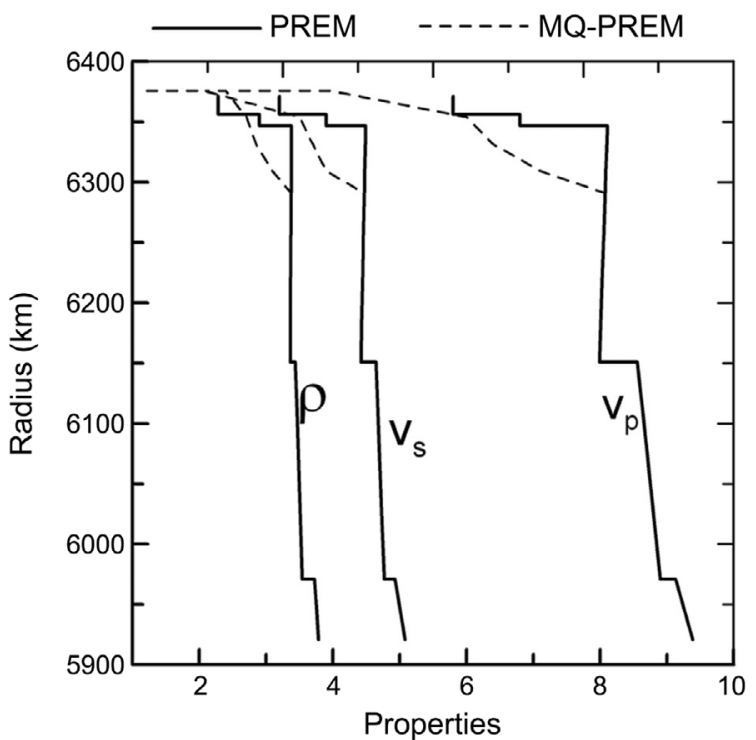

Fig. 1. Density and velocities for the Tibetan Plateau from PREM (solid line) and the modified version MQ-PREM with crustal structure replaced by averaged results (dashed line) from Crust2.0 (Laske et al. 2011). $\rho$ : density; $\mathrm{V}_{\mathrm{p}}: \mathrm{P}$ wave velocity; $\mathrm{V}_{\mathrm{s}}: \mathrm{S}$ wave velocity. (a)
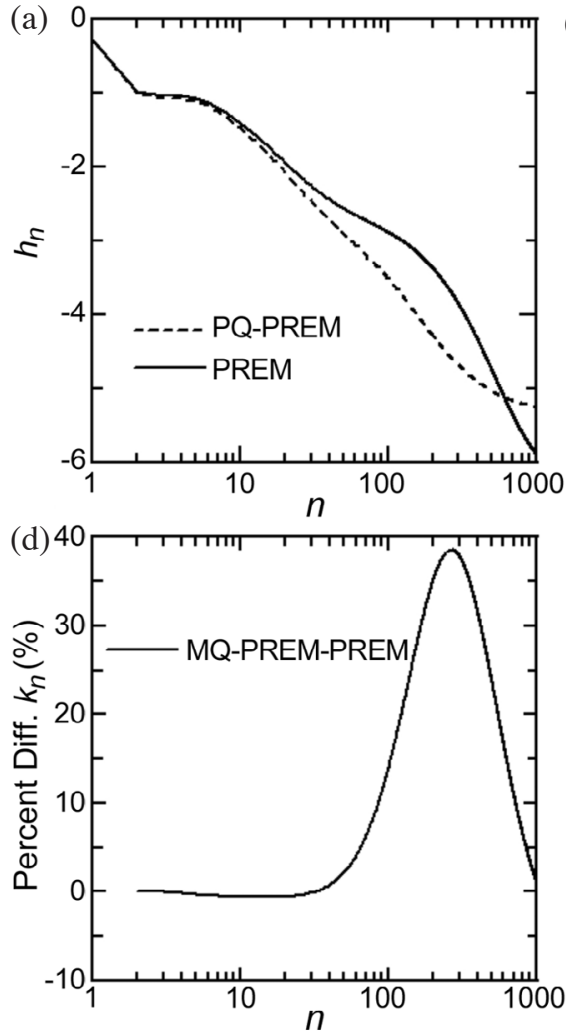

(b)

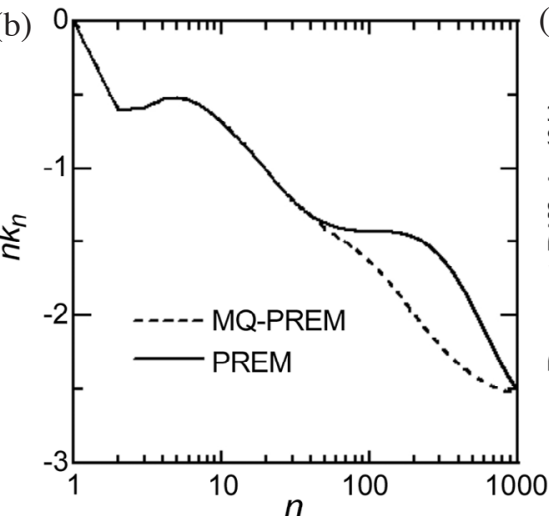

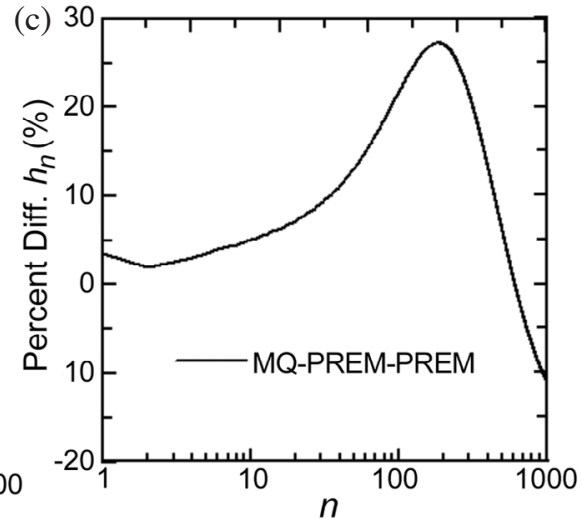

Fig. 2. Values of load Love numbers $h_{n}$ (a) and $k_{n}$ (b) for the MQPREM and PREM Earth models, and the differences between two Earth models in (c) and (d), respectively. 
expected, the results for the MQ-PREM model are larger than that for the PREM model with a maximum difference occurring at about degree 200. For the current situation, the GRACE time variable gravity field is available only up to degree 60 , and continuous GPS sites are very sparsely distributed in the Plateau. Thus the effect of the Earth model is small ( $<14 \%$ for $h_{n}$ and $<5 \%$ for $k_{n}$ ). However, in the near future, when the follow-on missions of GRACE and new continuous GPS measurements will have a much higher spatial resolution than the present, the maximum degrees needed may be much higher. For the maximum degrees of 120 and 180 , the relative differences are $24 \%$ and $28 \%$ for $h_{n}$, and $22 \%$ and $33 \%$ for $k_{n}$. So the higher the maximum degrees, the larger differences are found for the load Love numbers. The differences are so pronounced that the crustal structure may cause larger effects on the inversion of waters trend rates.

\section{METHODS}

In this section, we describe how we implement the forward computation for the simulated GRACE and GPS data using load Love numbers $\left(h_{l}, k_{l}\right)$ based on MQ-PREM including the Tibetan average crustal structure, and the simulated inversion for surface mass density using load Love numbers $\left(h_{l}^{\prime}, k_{l}^{\prime}\right)$ based on PREM. $\left(h_{l}, h_{l}^{\prime}\right)$ are load Love numbers for radial displacement and $\left(k_{l}, k_{l}^{\prime}\right)$ for potential perturbation, and the results can be found in Figs. $2 \mathrm{a}$ and $\mathrm{b}$.

\subsection{Forward Computation}

In order to produce simulated data for GRACE and GPS measurements, the forward computations have to be formulated.

Given the surface mass density of the EWT changes (or EWT trend rates) at any colatitude $\theta$ and longitude $\phi$ be $\sigma(\theta, \phi)$, then it can be decomposed into harmonics as:

$\sigma(\theta, \phi)=\sum_{l=0}^{M} \sum_{m=0}^{l}\left(c_{l m} \cos m \phi+s_{l m} \sin m \phi\right) \widetilde{P}_{l m}(\cos \theta)$

where $M$ is the maximum degree with $M \rightarrow \infty$ theoretically.

$$
\begin{aligned}
& \left\{\begin{array}{l}
c_{l m}=\frac{1}{4 \pi} \int \bar{R}_{l m}\left(\theta^{\prime}, \phi^{\prime}\right) \sigma\left(\theta^{\prime}, \phi^{\prime}\right) d \Omega^{\prime} \\
s_{l m}=\frac{1}{4 \pi} \int \bar{S}_{l m}\left(\theta^{\prime}, \phi^{\prime}\right) \sigma\left(\theta^{\prime}, \phi^{\prime}\right) d \Omega^{\prime}
\end{array}\right. \\
& \left\{\begin{array}{l}
\bar{R}_{l m}\left(\theta^{\prime}, \phi^{\prime}\right)=\widetilde{P}_{l m}\left(\cos \theta^{\prime}\right) \cos m \phi^{\prime} \\
\bar{S}_{l m}\left(\theta^{\prime}, \phi^{\prime}\right)=\widetilde{P}_{l m}\left(\cos \theta^{\prime}\right) \sin m \phi^{\prime}
\end{array}\right.
\end{aligned}
$$

$d \Omega^{\prime}=\sin \theta^{\prime} d \theta^{\prime} d \phi^{\prime}$, and $\widetilde{P}_{l m}$ is the normalized associated
Legendre polynomial.

When this surface mass is placed on the Earth, it deforms the Earth's surface and causes gravity perturbation. The gravity and displacement responses are given by the convolution of the load $\sigma(\theta, \phi)$ with the load Green's functions for gravity perturbation and radial displacement, which are (Farrell 1972) :

$\left\{\begin{array}{l}\delta g(\gamma)=\frac{g}{M_{e}} \sum_{l=0}^{M}(l+1)\left(1+k_{l}\right) P_{l}(\cos \gamma) \\ u_{r}(\gamma)=\frac{a}{M_{e}} \sum_{l=0}^{M} h_{l} P_{l}(\cos \gamma)\end{array}\right.$

Here, $h_{l}, k_{l}$ are the load Love numbers for radial displacement and potential perturbation based on MQ-PREM, $a$ is the earth's radius, $g$ is the surface gravity of the Earth, $G$ is the Newtonian gravitational constant, $M_{e}$ is the total mass of the Earth, $\gamma$ is the angular distance between observation site $(\theta, \phi)$ and the unit point mass located at $\left(\theta^{\prime}, \phi^{\prime}\right)$, and $P_{l}(\cos \gamma)$ is the Legendre polynomial. As discussed above, the load Love numbers depend upon the elastic structure of the Earth model, and are computed in the last section based on the Earth model MQ-PREM.

Convoluting the load given by Eq. (1) and the two Green's functions by Eq. (4) respectively gives the simulated gravity perturbation and radial displacement,

$\left\{\begin{array}{l}\delta g(\theta, \phi)=\frac{g a^{2}}{M_{e}} \int \sigma\left(\theta^{\prime}, \phi^{\prime}\right) \sum_{l=0}^{M}(l+1)\left(1+k_{l}\right) P_{l}(\cos \gamma) d \Omega^{\prime} \\ u_{r}(\theta, \phi)=\frac{a^{3}}{M_{e}} \int \sigma\left(\theta^{\prime}, \phi^{\prime}\right) \sum_{l=0}^{M} h_{l} P_{l}(\cos \gamma) d \Omega^{\prime}\end{array}\right.$

Using Eq. (2) and the addition theorem below:

$$
\begin{aligned}
& P_{l}(\cos \gamma)=\frac{1}{2 l+1} \\
& \times \sum_{m=0}^{l}\left[\bar{R}_{l m}\left(\theta^{\prime}, \phi^{\prime}\right) \bar{R}_{l m}(\theta, \phi)+\bar{S}_{l m}\left(\theta^{\prime}, \phi^{\prime}\right) \bar{S}_{l m}(\theta, \phi)\right]
\end{aligned}
$$

Eq. (5) becomes,

$$
\left\{\begin{aligned}
\delta g(\theta, \phi)= & 4 \pi G \sum_{l=0}^{M} \frac{l+1}{2 l+1}\left(1+k_{l}\right) \\
& \times \sum_{m=0}^{l}\left(c_{l m} \cos m \phi+s_{l m} \sin m \phi\right) \widetilde{P}_{l m}(\cos \theta) \\
u_{r}(\theta, \phi)= & \frac{3}{\bar{\rho}} \sum_{l=0}^{M} \frac{h_{l}}{2 l+1} \\
& \times \sum_{m=0}^{l}\left(c_{l m} \cos m \phi+s_{l m} \sin m \phi\right) \tilde{P}_{l m}(\cos \theta)
\end{aligned}\right.
$$

where $\bar{\rho}$ is the average density of the Earth.

Eq. (7) can be used to simulate the gravity perturbation and the vertical displacement observed by GRACE and 
GPS respectively. Note that the load Love numbers used in Eq. (7) are given from the realistic MQ-PREM.

\subsection{Simulated Inversion for Surface Mass Density}

Supposing that the GRACE gravity perturbation data and GPS vertical displacement data are available from the forward computation using Eq. (7), based on the two types of the simulated data, the surface mass density can be respectively inverted using the following formulas:

$$
\left\{\begin{aligned}
\sigma^{G R A C E}( & \theta, \phi)=\frac{1}{4 \pi G} \sum_{l=0}^{M} \sum_{m=0}^{l} \frac{2 l+1}{l+1} \frac{1}{1+k_{l}^{\prime}} \\
& \times\left(c_{l m}^{G R A C E} \cos m \phi+s_{l m}^{G R A C E} \sin m \phi\right) \tilde{P}_{l m}(\cos \theta) \\
\sigma^{G P S}(\theta, & \phi)=\frac{\rho}{3} \sum_{l=0}^{M} \sum_{m=0}^{l} \frac{2 l+1}{h_{l}^{\prime}} \\
& \times\left(c_{l m}^{G P S} \cos m \phi+s_{l m}^{G P S} \sin m \phi\right) \tilde{P}(\cos \theta)
\end{aligned}\right.
$$

where $\left(c_{l m}^{G R A C E}, s_{l m}^{G R A C E}\right)$ and $\left(c_{l m}^{G P S}, s_{l m}^{G P S}\right)$ are the coefficients for the spherical harmonic expansion similar to those in Eq. (1) but for the gravity perturbation data from GRACE and the vertical displacement data from GPS measurement respectively, and $h_{l}^{\prime}, k_{l}^{\prime}$ are the load Love numbers for radial displacement and potential perturbation given in the last section based on PREM.

Since the coefficients in Eq. (8) are already given in Eq. (7), we have the final formulas for the inverted surface mass density of EWT changes or EWT trend rates using the simulated GRACE and GPS data respectively,

$$
\left\{\begin{array}{l}
\sigma^{G R A C E}(\theta, \phi)=\sum_{l=0}^{M} \sum_{m=0}^{l} \frac{1+k_{l}}{1+k_{l}^{\prime}}\left(c_{l m} \cos m \phi+s_{l m} \sin m \phi\right) \tilde{P}_{l m}(\cos \theta) \\
\sigma^{G P S}(\theta, \phi)=\sum_{l=0}^{M} \sum_{m=0}^{l} \frac{h_{l}}{h_{l}^{\prime}}\left(c_{l m} \cos m \phi+s_{l m} \sin m \phi\right) \widetilde{P}(\cos \theta)
\end{array}\right.
$$

Accordingly, we can choose a hydrological model to compute the harmonic coefficients of the relevant surface mass density of the EWT changes (or EWT trend rates) using Eq. (2), the load Love numbers from a realistic Earth model MQ-PREM and PREM are respectively used for the data simulation and the simulated inversion, then the inverted surface mass density can be computed using Eq. (9) based on the two types of the simulated data.

\section{DATA}

We simulate GRACE and GPS data that only contain a pure hydrological signal. There are several hydrological models available, however, the WaterGap Global Hydrology Model (WGHM, Döll et al. 2003) appears to be a more sophisticated model since it takes into account major hydrological processes such as snow accumulation and melting, evapo-transpiration, runoff generation, lateral transport of water, and the data are continuously updated. Thus, we employ WGHM in the Tibetan Plateau to simulate the hydrological GRACE and GPS signal from August 2002 to March 2011.

Using 0.5 deg. $\times 0.5$ deg. grid data of WGHM, we derive the secular trend rates of EWT changes and decompose them into harmonics using Eq. (2), and then do a synthesis computation as in Eq. (1) for three maximum degrees $M=$ 60, 120 and 180 which are shown in Figs. 3a, b and c, respectively. It can be seen that larger water rise trends appear

(a)
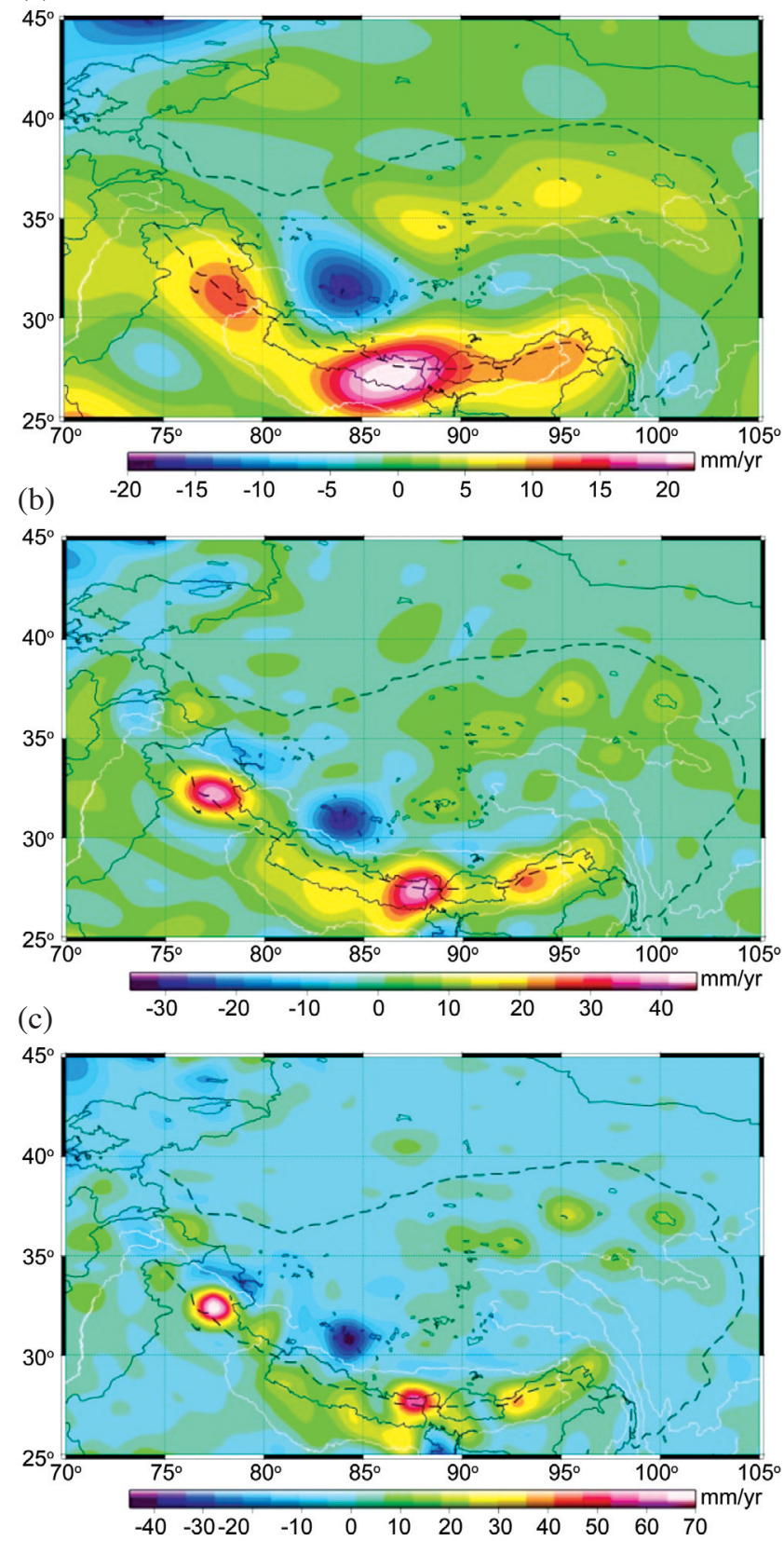

Fig. 3. EWT trend rates [mm yr-1] from August 2002 to March 2011 from WGHM developed into spherical harmonics up to degrees (a) 60 , (b) 120 , and (c) 180 . Note the different scales for the subfigures. 
along the Himalaya mountain range and in the northern part of the Plateau. The higher the maximum degree for synthesis is, the larger magnitudes for the EWT trends result.

Using Eq. (7) with the load Love numbers $h_{l}, k_{l}$ given by Figs. $2 \mathrm{a}$ and $\mathrm{b}$, we simulate the trend rates of gravity perturbation and vertical displacement shown in Fig. 4. It is found that GRACE and GPS data are reasonably simulated since the gravity is increasing and the crust is subsiding in the areas where the water is rising based on the computational results.

\section{RESULTS}

In the foregoing, the simulated data are calculated with load Love numbers $h_{l}$ and $k_{l}$ from MQ-PREM. In addition, we simulate inversions using Eq. (9) for EWT trend rates (a)
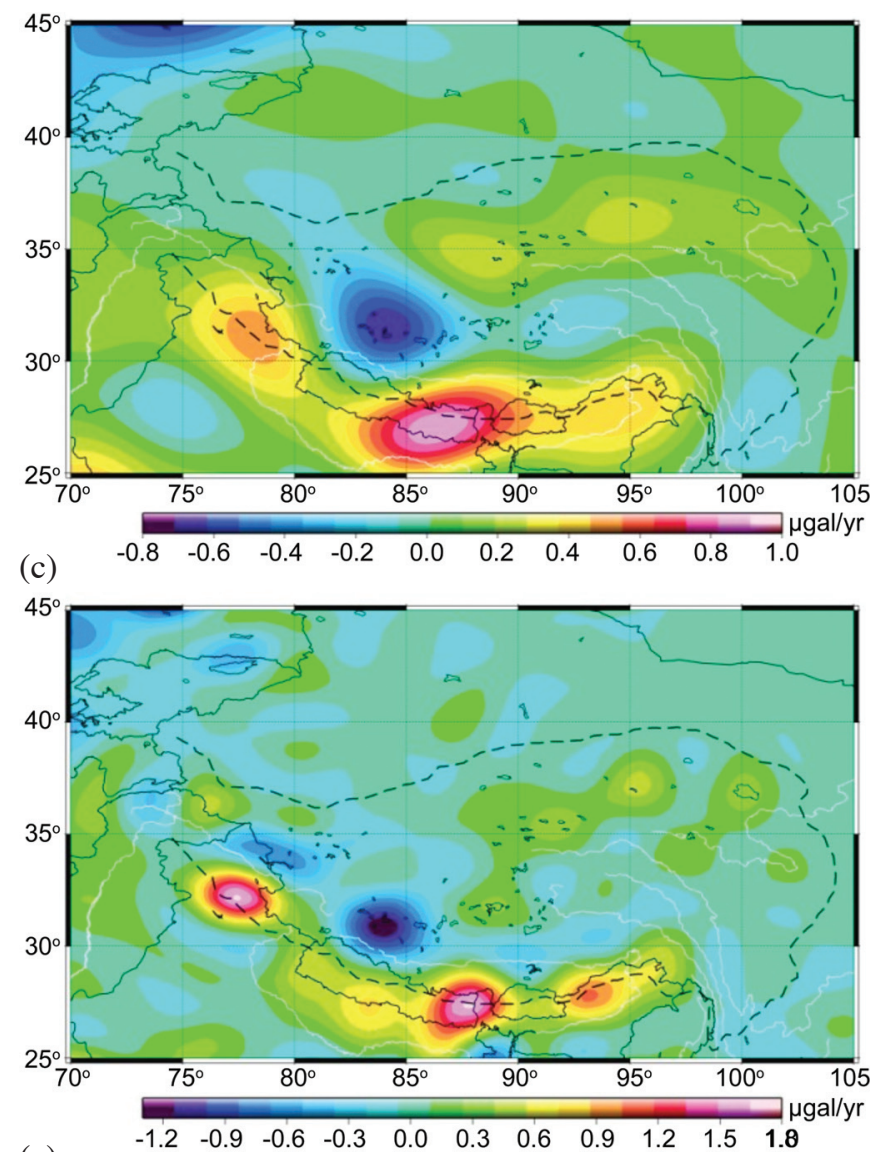

(e)

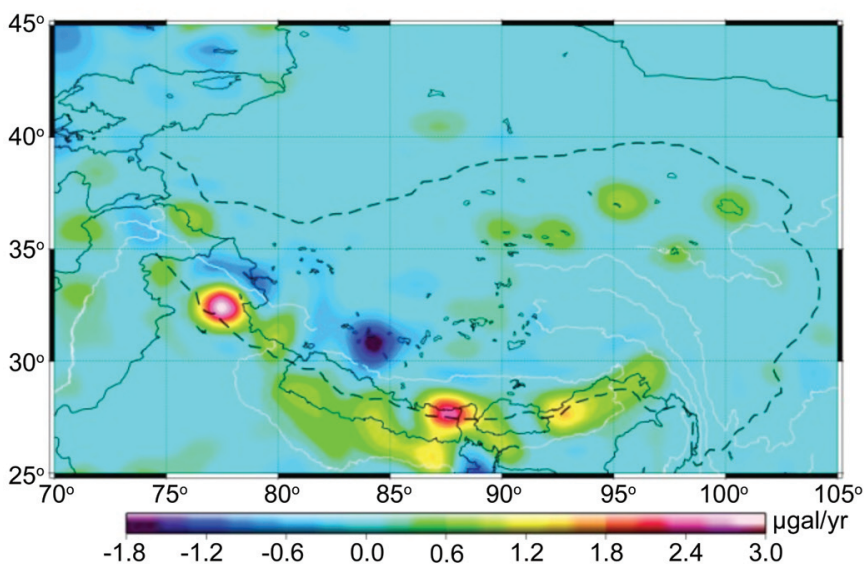

(b)

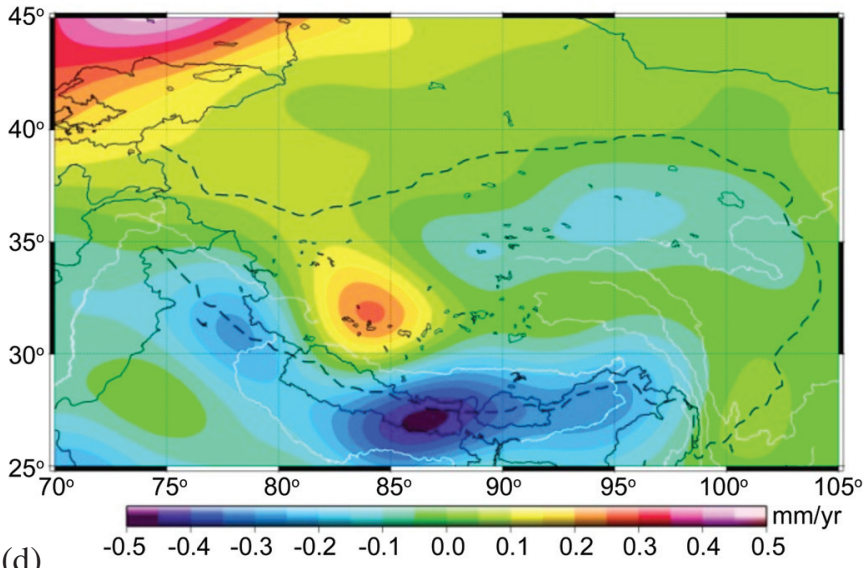

(d)

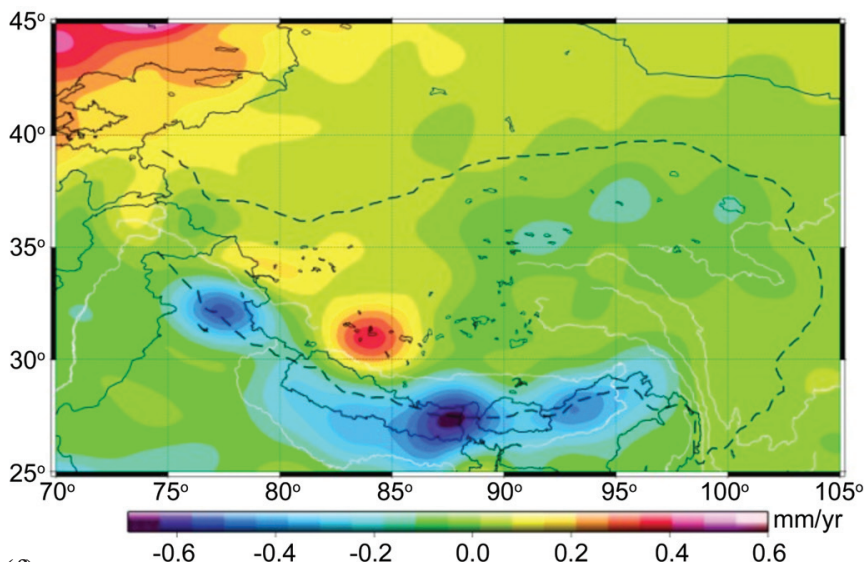

(f)

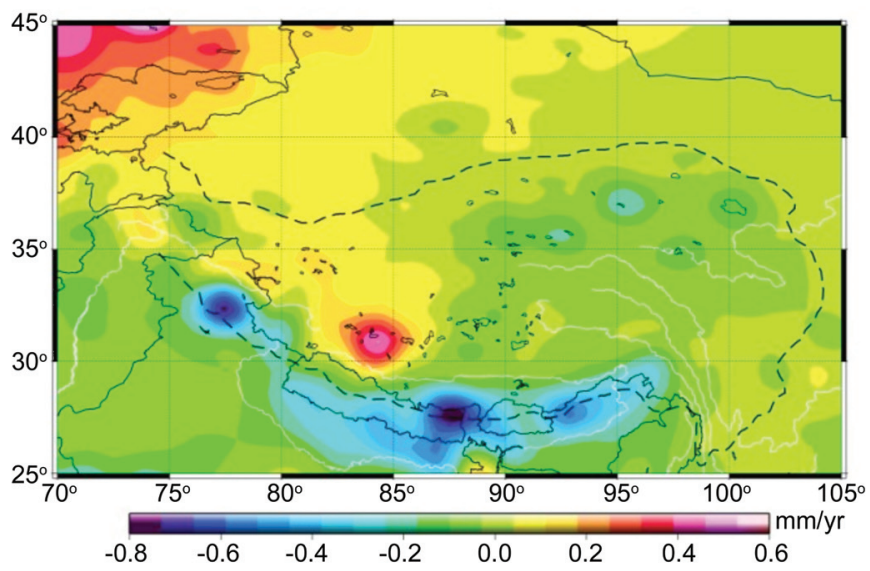

Fig. 4. Simulated hydrological contribution in GRACE (left) $\left[\mu \mathrm{Gal} \mathrm{yr}^{-1}\right]$ and GPS (right) data $\left[\mathrm{mm} \mathrm{yr}^{-1}\right]$ using WGHM. (a), (c), and (e) are the trend rates of gravity perturbation with spherical harmonics up to degrees 60,120, and 180, respectively. (b), (d), and (f) are the same as (a), (c), and (e), respectively, but for vertical displacement. 
based on simulated GRACE and GPS data in Fig. 4 but with Love numbers $h_{l}^{\prime}, k_{l}^{\prime}$ from PREM. Comparison of the inverted results with the simulated data gives the differences in Figs. 5, and 6.

In Fig. 5, we show the effects of crustal differences in the Tibetan Plateau on inversion of EWT trend rates when simulated GRACE data are used. For degree 60 which marks the current resolution of provided Release 4 GRACE data, the inverted results are presented in Fig. 5a. The differences between using MQ-PREM and PREM (Fig. 5b) are very small with a magnitude of at most $0.01 \mathrm{~mm} \mathrm{yr}^{-1}$. Figures $5 \mathrm{c}$, $\mathrm{d}$ a, e, and $\mathrm{f}$ highlight the results for truncated degrees 120 and 180. The inversion results (Figs. 5c, e) are still quite very similar to the values (Figs. 3b, c) given by WGHM. However, the differences become larger with magnitudes of $\sim 0.04$ and $\sim 0.06 \mathrm{~mm} \mathrm{yr}^{-1}$, respectively, when increasing the maximum degree $M$. Thus, the effects of crustal differences in the Tibetan Plateau on inversion of EWT trend rates are (a)
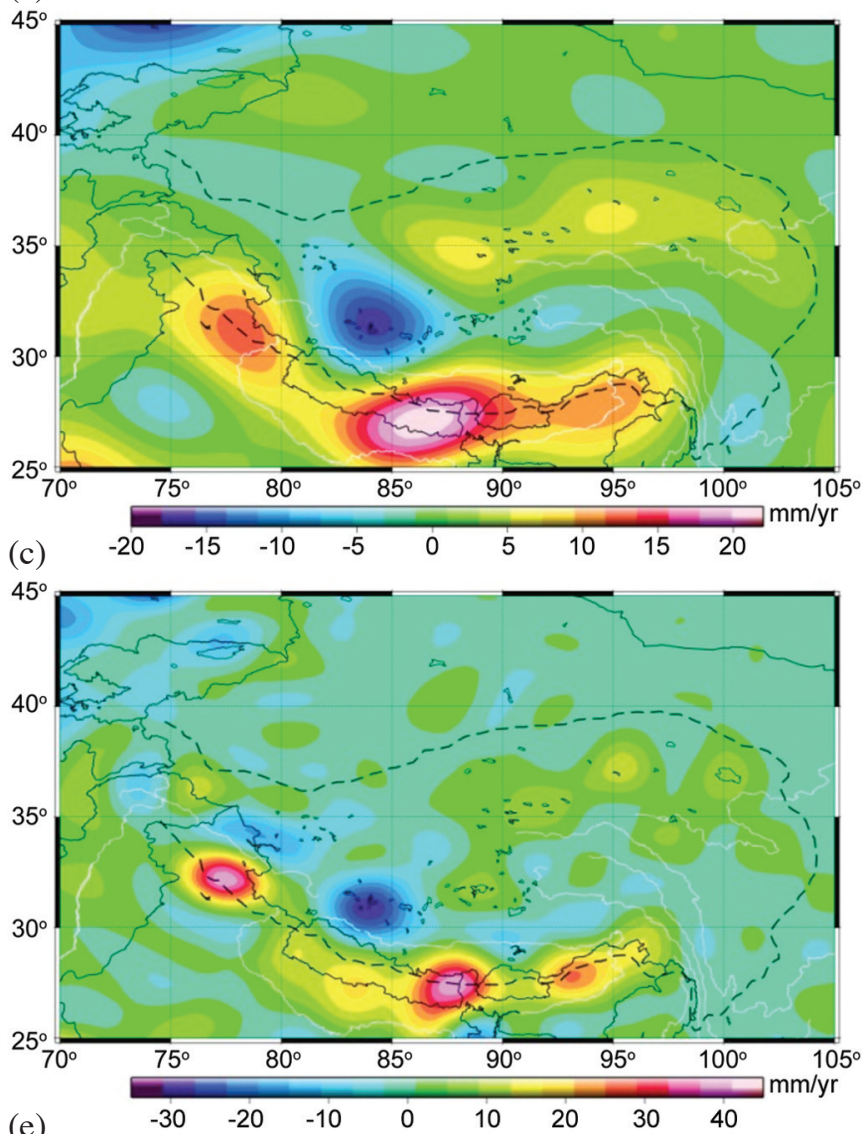

(e)

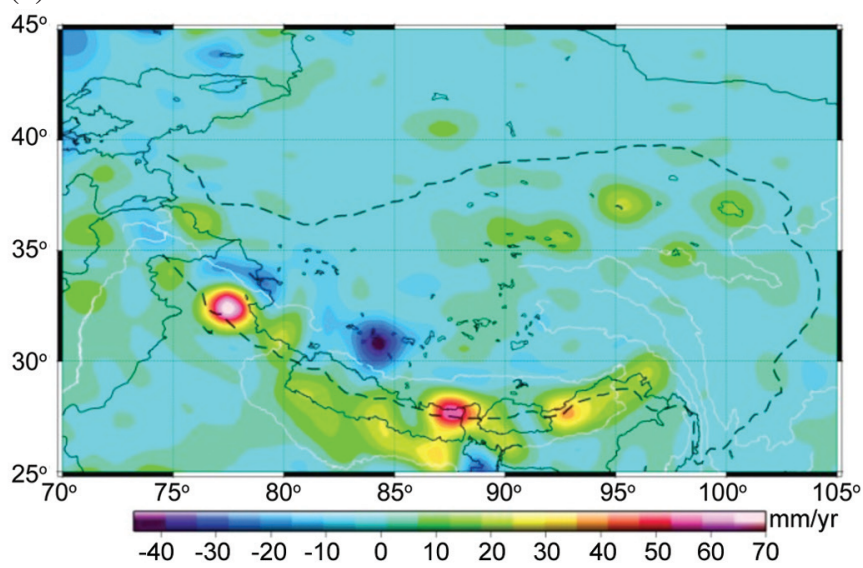

(b)

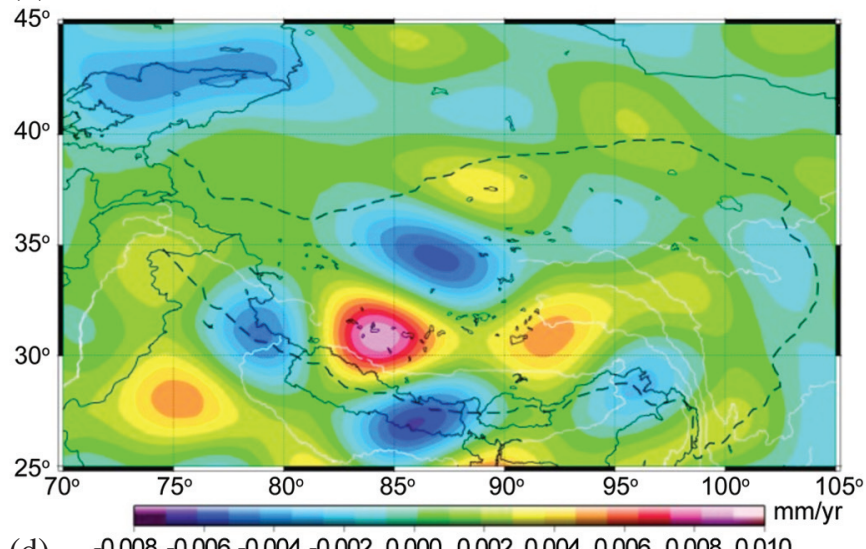

(d)

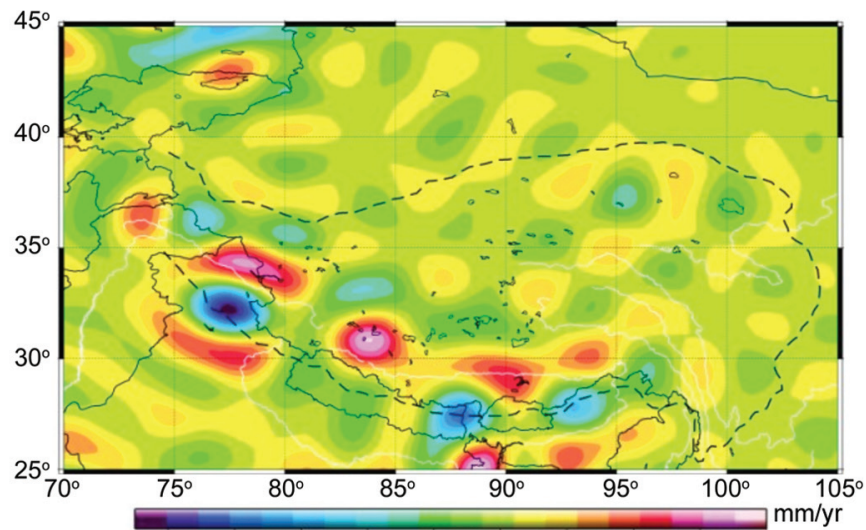

(f)

$\begin{array}{llllllllll}-0.05 & -0.04 & -0.03 & -0.02 & -0.01 & 0.00 & 0.01 & 0.02 & 0.03 & 0.04\end{array}$

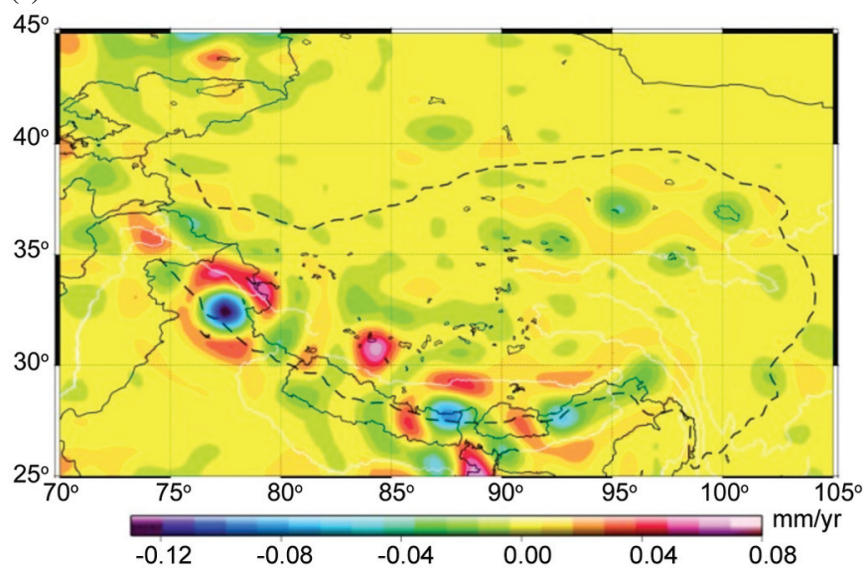

Fig. 5. EWT trend rates inverted from simulated trends of GRACE gravity perturbation. (a), (c), and (e) are the results of the simulated inversion with spherical harmonics up to degrees 60, 120, and 180, respectively. (b), (d), and (f) are corresponding differences to WGHM in Fig. 3a, b, and c. 
generally small. The reason is that the gravity signals are dominated by the direct attraction of the surface mass load and only partially affected by load induced deformation term as apparent in the first formula of Eq. (7). Therefore, although the differences of load Love number between $k_{l}^{\prime}$ and $k_{l}$ are quite pronounced in Figs. $2 \mathrm{~b}$ and d choosing MQPREM instead of PREM would not change the inversion results too much.

When simulated GPS data are used, larger effects of crustal differences in the Tibetan Plateau on inversion of EWT trend rates are found (Fig. 6). First, when checking the second formula of Eq. (7), we see that the GPS signals are totally dependent upon the loading term with load Love number $h_{l}^{\prime}$, thus any difference in the load Love number values of MQ-PREM and PREM would affect the results of the simulated inversion. The differences (Figs. 6b, d, f) with respect to those given by WGHM (Figs. 3a, b, c) are large with magnitudes of $\sim 2.0, \sim 5.0$, and $\sim 15.0 \mathrm{~mm} \mathrm{yr}^{-1}$. (a)
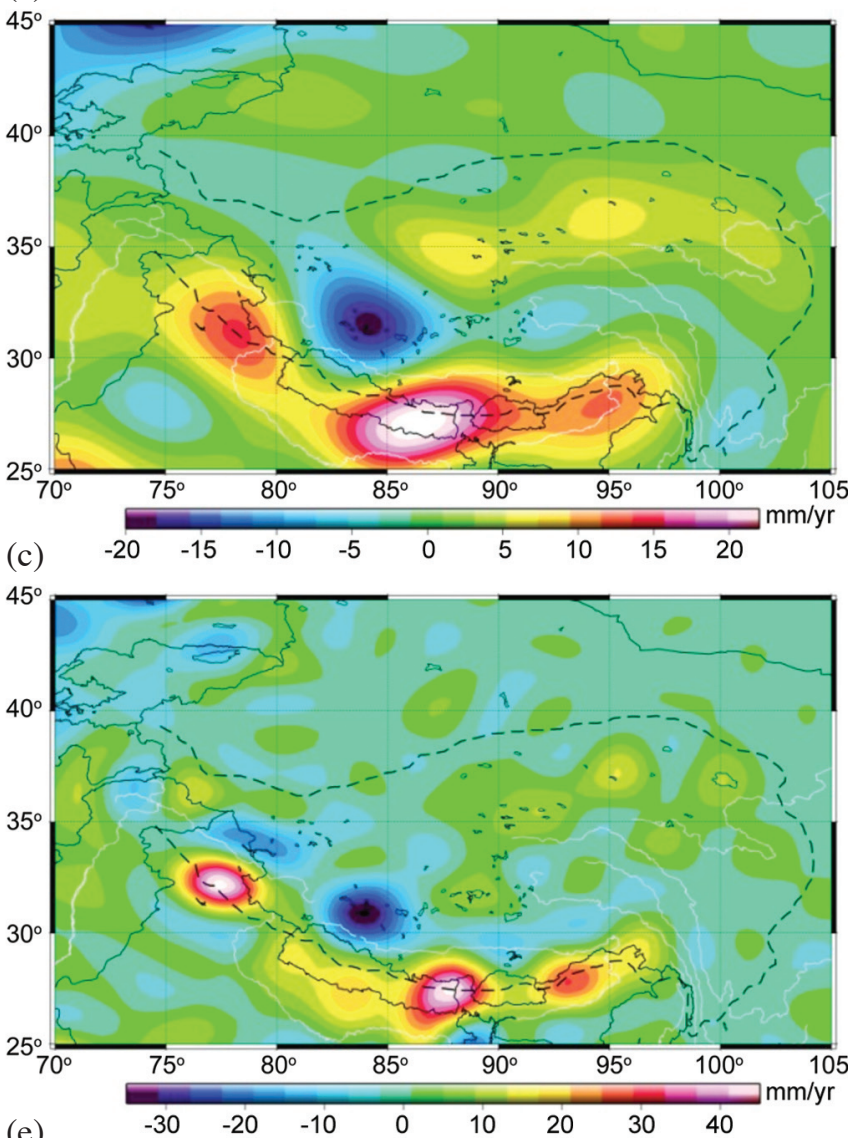

(e)

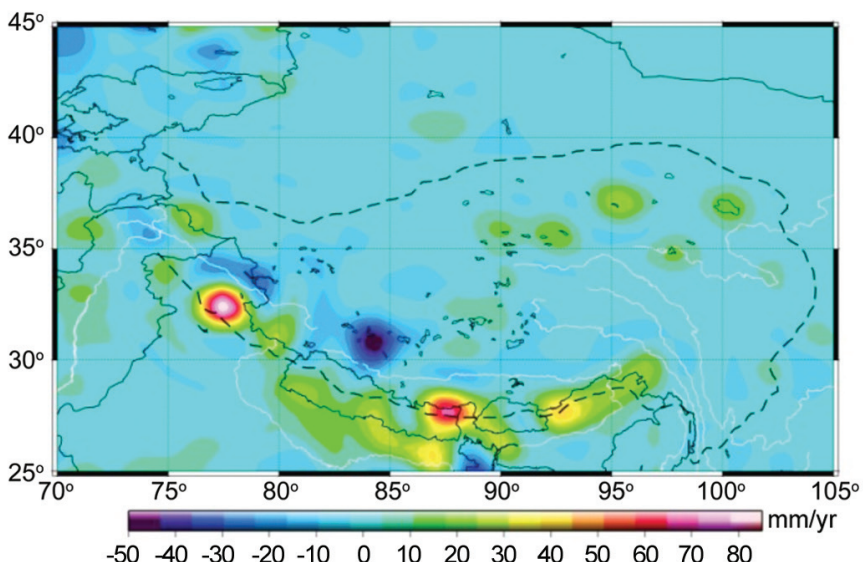

(b)
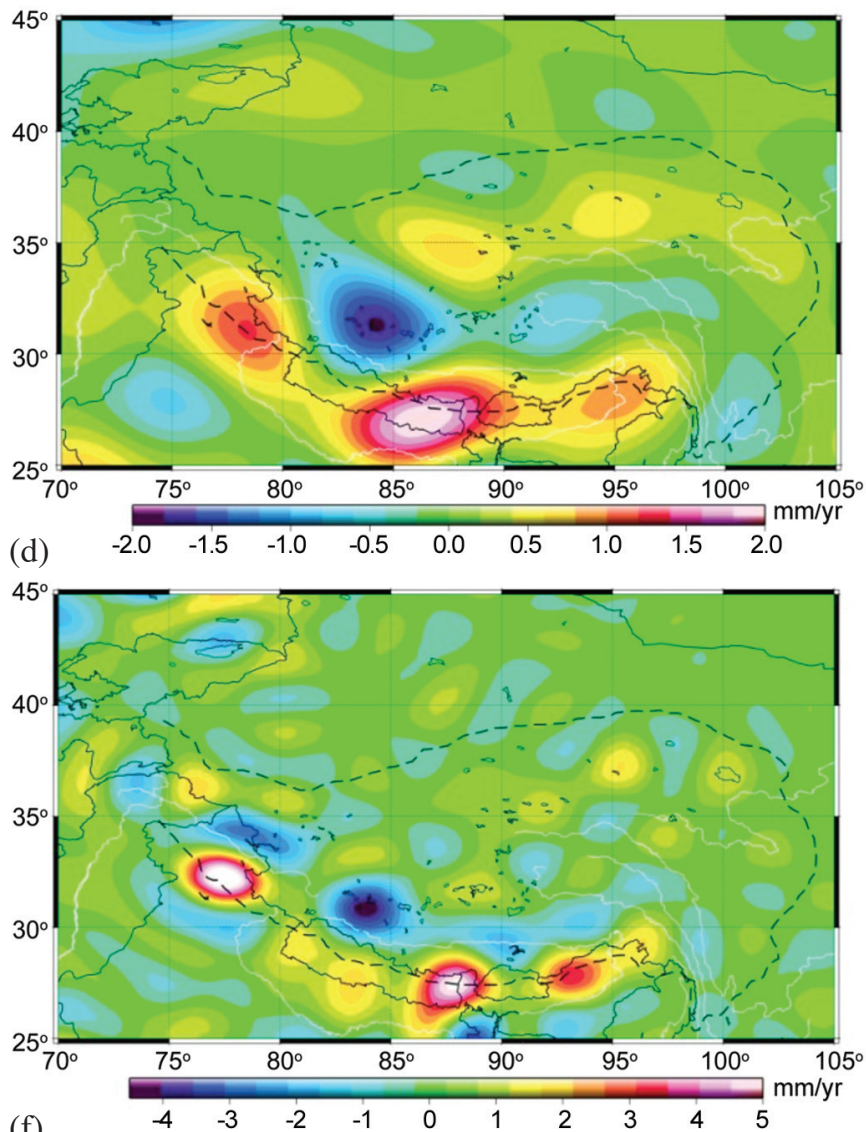

(f)

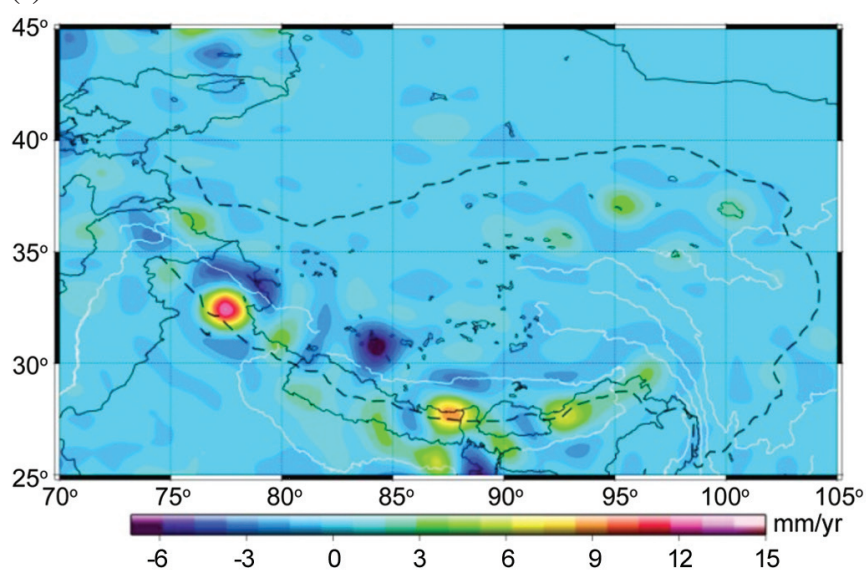

Fig. 6. EWT trend rates inverted from simulated trends of GPS vertical displacement. (a), (c), and (e) are the results of the simulated inversion with harmonics up to degrees 60,120, and 180, respectively. (b), (d), and (f) are corresponding differences to WGHM in Figs. 3a, b, and c. 


\section{CONCLUSIONS}

Through the inversions of water trend rates in the $\mathrm{Ti}-$ betan Plateau using the simulated GRACE and GPS data, we studied the effects of two different crust models on the simulated inversion results. First, when using simulated GRACE or GPS data, the effects become larger with increasing maximum degree $M$ of the synthesis computation for simulated data. Thus, assuming that the resolution of GRACE and GPS data will increase in the near future, the effects on the inversion results with such data may impact future analyses and their results. However, we note also that when using simulated GRACE data for the inversion, the effects are negligible independent of the maximum degree. Therefore, PREM is suitable for the inversion of water trend rates in our investigation area and also anywhere else in the world based on present and even follow-on GRACE data. Third and in contrast to GRACE, when using simulated GPS data, the effects of crustal differences are very prominent with magnitudes of 2,5 , and $15 \mathrm{~mm} \mathrm{yr}^{-1}$ for maximum degrees of 60,120 and 180 , respectively. This means that PREM would not be applicable to the inversion of water trend rates in the Tibetan Plateau using GPS network data in the future. In this case a modified Earth model with a more realistic crustal structure such as MQ-PREM in this paper should be used.

Acknowledgements Hansheng Wang is supported by CAS/ SAFEA International Partnership Program for Creative Research Teams (Grant No. KZZD-EW-TZ-05), National Natural Science Foundation of China (Grant No. 40825012, 41021003, 41174016, 41274026) and National key Basic Research Program of China (973 Program, Grant Nos. 2012CB957703). Liming Jiang is supported by the Hundred Talents Program of the Chinese Academy of Sciences. We thank Andreas Guntner (GFZ Potsdam) for providing the WGHM data. We are grateful for the constructive comments by Dr. Junyi Guo and an anonymous reviewer. Some figures are drawn using the GMT graphics package (Wessel and Smith 1998).

\section{REFERENCES}

Chen, J. L., C. R. Wilson, D. Blankenship, and B. D. Tapley, 2009: Accelerated antarctic ice loss from satellite gravity measurements. Nat. Geosci., 2, 859-862, doi: 10.10 38/ngeo694. [Link]

Döll, P., F. Kaspar, and B. Lehner, 2003: A global hydrological model for deriving water availability indicators: Model tuning and validation. J.Hydrol., 270, 105134, doi: 10.1016/S0022-1694(02)00283-4. [Link]

Dziewonski, A. M. and D. L. Anderson, 1981: Preliminary reference Earth model. Phys. Earth Planet. Inter., 25, 297-356, doi: 10.1016/0031-9201(81)90046-7. [Link]

Farrell, W. E., 1972: Deformation of the Earth by surface loads. Rev. Geophys., 10, 761-797, doi: 10.1029/RG01 0i003p00761. [Link]

Guo J. Y., J. S. Ning, and F. P. Zhang, 2001: Chebyshevcollocation method applied to solve ODEs in geophysics singular at the Earth center. Geophys. Res. Lett., 28 , 3027-3030, doi: 10.1029/2001GL012886. [Link]

Han, D. and J. Wahr, 1995: The viscoelastic relaxation of a realistically stratified Earth, and a further analysis of postglacial rebound. Geophys. J. Int., 120, 287-311, doi: 10.1111/j.1365-246X.1995.tb01819.x. [Link]

Jacob, T., J. Wahr, W. T. Pfeffer, and S. Swenson, 2012: Recent contributions of glaciers and ice caps to sea level rise. Nature, 482, 514-518, doi: 10.1038/nature 10847 . [Link]

Laske, G., G. Masters, and C. Reif, 2011: A New Global Crustal Model at $2 \times 2$ Degrees. CRUST 2.0, available at http://igppweb.ucsd.edu/ gabi/crust2.html.

Leuliette, E. W. and L. Miller, 2009: Closing the sea level rise budget with altimetry, Argo, and GRACE. Geophys. Res. Lett., 36, L04608, doi: 10.1029/2008GL036010. [Link]

Matsuo, K. and K. Heki, 2010: Time-variable ice loss in Asian high mountains from satellite gravimetry. Earth Planet. Sci. Lett., 290, 30-36, doi: 10.1016/j.epsl.2009. 11.053. [Link]

Rodell, M., I. Velicogna, and J. S. Famiglietti, 2009: Satellite-based estimates of groundwater depletion in India. Nature, 460, 999-1002, doi: 10.1038/nature08238. [Link]

Tapley, B. D., S. Bettadpur, J. C. Ries, P. F. Thompson, and M. M. Watkins, 2004: GRACE measurements of mass variability in the Earth System. Science, 305, 503-505, doi: 10.1126/science.1099192. [Link]

Van Dam, T., H.-P. Plag, O. Francis, and P. Gegout, 2002: GGFC Special Bureau for Loading: Current Status and Plans. IERS Technical Note, 30, 180-198.

Wahr, J., M. Molenaar, and F. Bryan, 1998: Time variability of the Earth's gravity field: Hydrological and oceanic effects and their possible detection using GRACE. J. Geophys. Res., 103, 30205-30229, doi: 10.1029/98 JB02844. [Link]

Wang, H., H. Hsu, and G. Li, 1996: Improvement of computations of load Love numbers of SNREI Earth model. Chin. J. Geophys., 39, 182-189. (in Chinese)

Wang, H., L. Xiang, L. Jia, L. Jiang, Z. Wang, B. Hu, and P. Gao, 2012: Load Love numbers and Green's functions for elastic Earth models PREM, iasp91, ak135, and modified models with refined crustal structure from Crust 2.0. Comput. Geosci., 49, 190-199, doi: 10.1016/j.cageo. 2012.06.022. [Link]

Wang, H., Z. Wang, X. Yuan, P. Wu, and E. Rangelova, 2007: Water storage changes in Three Gorges water systems area inferred from GRACE time-variable gravity data. Chin. J . Geophys., 50, 730-736. (in Chinese)

Wessel, P. and W. H. F. Smith, 1998: New, improved version of generic mapping tools released. Eos, Trans., $A G U, 79$, p 579, doi: 10.1029/98EO00426. [Link] 